\title{
Deficiência de ferro na insuficiência cardíaca
}

\section{Iron deficiency in heart failure patients}

Antonio Carlos P. Barretto ${ }^{1}$

Milena N. Cardoso ${ }^{2}$

Juliano N. Cardoso ${ }^{3}$

\begin{abstract}
A anemia é uma comorbidade frequente nos pacientes com insuficiência cardíaca (IC) e sua presença parece estar associada à pior evolução, sendo descrita em alguns estudos como fator de prognóstico independente tanto na IC sistólica quanto na diastólica. Entretanto, ainda não sabemos se a anemia causa pior evolução ou se é apenas um marcador do maior comprometimento cardíaco nos portadores de IC. A etiologia da anemia na IC é multifatorial e parece variar conforme a população estudada. Os fatores como a deficiência nutricional de ferro, presença de insuficiência renal, intensa atividade inflamatória sistêmica, uso de medicações que inibem a produção de eritropoetina ou que causam perda sanguínea são os mais frequentes causadores de anemia na IC. A prevalência de anemia citada nos estudos de IC é muito variável (de $9 \%$ a 79,1\%) e isto é dependente da população estudada, da fase da cardiopatia, do método e referências hematimétricas utilizadas para diagnóstico. A deficiência de ferro é um importante fator etiológico e está presente em um número significativo de pacientes com anemia e IC associada. Em estudo realizado em nosso grupo, a incidência de deficiência de ferro nos pacientes com anemia foi de 61,8\%. Portanto, a anemia é um achado frequente, sua presença acentua as manifestações clínicas da IC e está associada a piora do prognóstico. Conhecer a causa da anemia facilita o seu tratamento e, apesar da sua correção ainda não ser consenso, os pacientes sem anemia têm melhor evolução. Rev. Bras. Hematol. Hemoter. 2010; 32(Supl.2):89-94.
\end{abstract}

Palavras-chave: Insuficiência cardiaca; anemia; deficiência de ferro.

\section{Introdução e prevalência}

A insuficiência cardíaca (IC) é uma síndrome em que o coração apresenta sua capacidade de enchimento ou ejeção dos ventrículos diminuída, resultando na incapacidade de suprir as necessidades metabólicas dos tecidos. ${ }^{1,2}$ É a fase final comum das doenças cardíacas, sendo causa frequente de seguimento ambulatorial, de internação hospitalar e ainda responsável por altas taxas de morbidade e mortalidade., ${ }^{3,4}$ Antigamente, apenas hemoglobina abaixo de 9,0 g/dL era valorizada; atualmente, sabemos que qualquer grau de anemia pode piorar a evolução do doente portador de IC. A anemia é uma comorbidade frequente nos pacientes com IC e sua presença está associada à pior evolução, sendo descrita em alguns estudos como fator de prognóstico independente. ${ }^{5,6}$ Entretanto, não sabemos, até o presente momento, se a anemia causa uma pior evolução ou se é apenas um marcador de maior comprometimento cardíaco e sistêmico dos pacientes.

A etiologia da anemia na IC é considerada multifatorial, podendo ser causada pelo volume plasmático aumentado (hemodiluição) ou pela diminuição da massa de células vermelhas (anemia verdadeira). ${ }^{2}$ Podemos citar alguns fatores que contribuem para a redução da hemoglobina, como a

${ }^{1}$ Cardiologista. Professor Associado da USP, diretor do Serviço de Prevenção e Reabilitação do InCor - FMUSP - São Paulo-SP.

${ }^{2}$ Cardiologista. Médica Estagiária do Serviço de Cardiologia do InCor - FMUSP - São Paulo-SP.

${ }^{3}$ Cardiologista. Médico Assistente HÁ Cotoxó - InCor - FMUSP; Coordenador da Cardiologia do Hospital Santa Marcelina - São Paulo-SP.

Hospital de Cotoxó - InCor - FMUSP - São Paulo-SP.

Correspondência: Antonio Carlos Pereira Barretto

Rua Cotoxó, 1142

05012-000 - São Paulo-SP - Brasil

Tel.: (55 11) 3879-2310

E-mail: pereira.barretto@incor.usp.br

Doi: 10.1590/S1516-84842010005000053 
deficiência de ferro, ácido fólico e vitamina B12 por baixa ingestão, má absorção ou perda crônica; alteração no metabolismo de ferro; ${ }^{7}$ deficiência na produção de eritropoetina secundária a insuficiência renal; ${ }^{8}$ aumento das citocinas (principalmente do fator de necrose tumoral alfa), que reduzem a produção de eritropoetina, inibem a hematopoese e reduzem a disponibilidade de ferro para utilização pela medula óssea; ${ }^{8}$ uso de fármacos, como os inibidores da enzima conversora de angiotensina (IECA), os bloqueadores dos receptores de angiotensina I (BRA) e o carvedilol, que também inibem a produção de eritropoetina. ${ }^{9}$

Estudos revelam prevalência de anemia muito variável ( $9 \%$ a $79,1 \%$ ) nos portadores de IC, e isto é dependente da população estudada, da fase da cardiopatia, do método e referências para os valores de hemoglobina $(\mathrm{Hb})$ e hematócrito (Ht) utilizados para diagnóstico. ${ }^{10-15}$ Em estudo que analisou pacientes com disfunção do ventrículo esquerdo, foi encontrada prevalência de anemia de $22 \%$, quando definiu como referência Hematócrito $<39 \%$ e $\mathrm{Hb}<13 \mathrm{~g} / \mathrm{dL}$, porém, ao definir como $\mathrm{Ht}<35 \%$ e $\mathrm{Hb}<12 \mathrm{~g} / \mathrm{dL}$ foi encontrada prevalência de apenas $4 \% .{ }^{16}$ A Organização Mundial de Saúde define anemia nos homens para valores de hemoglobina $<13,0 \mathrm{~g} / \mathrm{dL}$ e nas mulheres $<12 \mathrm{~g} / \mathrm{dL}$, mas até o momento não há dados que permitam indicar qual o número de hemoglobina deve ser utilizado como referência nos pacientes com IC. A prevalência da anemia em pacientes com IC em classe funcional (CF) IV da New York Heart Association (NYHA), refratários ao tratamento medicamentoso, aproxima de $80 \%$ em alguns estudos, enquanto em pacientes em CF I ou II de NYHAé de $10 \% \cdot{ }^{17,14}$ Além do grau de acometimento cardíaco, outros fatores estão associados a um aumento de prevalência da anemia na IC, como a insuficiência renal, idade avançada, sexo feminino e descendentes africanos. ${ }^{17,18}$

A anemia é tão prevalente na IC sistólica quanto na diastólica. ${ }^{18}$ Felker et al., em um estudo retrospectivo, incluindo 4.951 pacientes com insuficiência cardíaca sintomática (3.093 com disfunção diastólica e 1.858 com disfunção sistólica), demonstraram uma prevalência de anemia $(\mathrm{Hb}<13$ em homens e $\mathrm{Hb}<12$ em mulheres) de $38 \%$ em pacientes com disfunção diastólica e de $41 \%$ em pacientes com disfunção sistólica. ${ }^{18}$

A deficiência de ferro ( $\mathrm{Fe}$ ) é uma causa importante em pacientes com insuficiência cardíaca e apresenta múltiplas causas, como ingestão deficiente deste elemento, má absorção devido ao edema da alças intestinais, perda sanguínea pelo trato gastrintestinal causada por medicações anticoagulantes e antiplaquetárias, gastrites urêmicas e alteração no transporte de Fe causado pelo aumento da atividade de citocinas. ${ }^{19}$ A prevalência da anemia ferropriva na IC varia entre os estudos e isto depende do método usado para diagnóstico. Nanas et al. investigaram causas de anemia e, utilizando como método a aspiração e coloração de medula óssea, encontraram deficiência de ferro em $73 \%$ dos 37 pacientes hospitalizados com IC avançada. ${ }^{19}$
Em estudo realizado em nosso hospital (Hospital de Cotoxó - InCor) no ano de 2007, foram avaliados pacientes que internaram devido a IC descompensada e fração de ejeção do ventrículo esquerdo (FEVE) $\leq 45 \%$. A incidência de anemia (definida como valores de $\mathrm{Hb}<12 \mathrm{~g} / \mathrm{dL}$ para ambos os sexos) nesta série de casos foi de $34,3 \%$. Deficiência de ferro (ferritina sérica menor que $100 \mathrm{ng} / \mathrm{mL}$ e/ou saturação de transferrina menor que 20\%) foi encontrada em $61,8 \%$ dos pacientes anêmicos. Neste estudo, os pacientes anêmicos eram mais idosos, apresentavam função renal mais comprometida e níveis de BNP mais elevados em comparação aos não anêmicos, e a anemia foi um marcador independente de mau prognóstico. A mortalidade dos pacientes anêmicos foi maior do que a dos não anêmicos $(47 \%$ vs $24,6 \% \mathrm{p}=0,016$ Figura 1). Como causa da anemia, nesta população de pacientes com IC avançada, contribuem a desnutrição e a inapetência, que participam na gênese, a depleção de ferro encontrada em nossos pacientes, além dos fatores inflamatórios, com elevação de TNF-alfa, que inibem a absorção de Fe. Participam também, nesta gênese, as perdas sanguíneas relacionados ao uso de medicações como ácido acetilsalicílico.

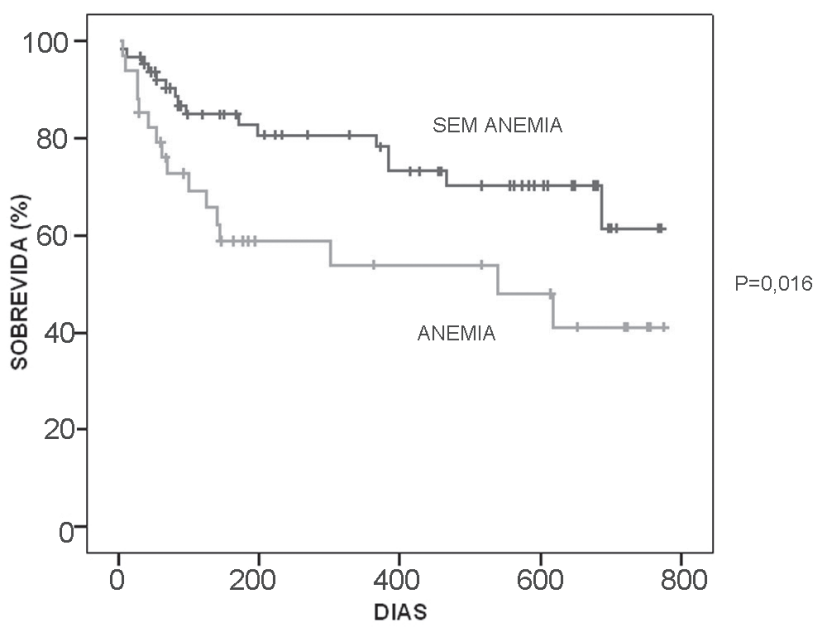

Figura 1. Curva de sobrevida durante o acompanhamento dos paciente com e sem anemia que internaram com IC sistólica

\section{Fisiopatologia da anemia na insuficiência cardíaca}

A anemia reduz a oferta de oxigênio $\left(\mathrm{O}_{2}\right)$ aos tecidos, causando uma sucessão de respostas neuro-humorais, cardiovasculares e renais. ${ }^{119} \mathrm{~A}$ anemia causa isquemia tissular e isto provoca vasodilatação periférica, diminuição da pressão arterial, ativação do sistema nervoso simpático, que, por sua vez, promove taquicardia reflexa e vasoconstrição generalizada, reduzindo o fluxo sanguíneo renal e ativando o SRAA (Sistema Renina Angiotensina Aldosterona). ${ }^{18-21}$ A angiotensina II promove vasoconstrição renal (artéria eferente) e sistêmica, aumenta a liberação de noradrenalina, inibe o tônus 
vagal, estimula a liberação de aldosterona e leva à disfunção endotelial. Como consequência há retenção de sódio e água e excreção de potássio. Inicialmente, estes mecanismos são adaptativos, porém, a ativação neuro-humoral prolongada leva à remodelação miocárdica e evolução da IC para uma fase mais avançada. ${ }^{1}$ Portanto, a anemia também contribui para a remodelação ventricular e progressão da doença na IC devido ao estímulo neuro-humoral, ativação de citocinas e promoção de hipertrofia ventricular esquerda. O efeito da anemia no remodelamento cardíaco tem sido avaliado em estudos de caráter experimental. Em ratos, a anemia induziu à hipertrofia excêntrica, aumento da proliferação de capilares e aumento de $50 \%$ da massa muscular cardíaca. ${ }^{20}$ A relação entre anemia e remodelamento cardíaco também tem sido tema de estudo em pacientes com insuficiência renal associada. Levin et al. observaram que, nos pacientes com insuficiência renal em fase inicial, ocorre uma diminuição da concentração de hemoglobina associada ao aumento de hipertrofia do ventrículo esquerdo (VE). ${ }^{21}$

Os pacientes com miocardiopatia isquêmica podem ter sua doença exacerbada pela anemia, devido à piora da hipóxia miocárdica e periférica; ${ }^{2}$ assim, os mecanismos da anemia se somam ao da insuficiência cardíaca, resultando em piora do quadro clínico e acentuação da cardiopatia. ${ }^{19}$

A anemia pode ser um marcador de maior comprometimento sistêmico dos pacientes, uma vez que o paciente com maior alteração cardíaca ou com doença mais prolongada apresenta inapetência, redução da perfusão renal e acentuada estimulação neuro-hormonal e das citocinas, todos os fatores reconhecidos como potenciais causadores de anemia.

\section{Repercussões clínicas}

O paciente portador de IC apresenta sintomas como dispneia, fadiga e intolerância ao esforço físico. Os sinais relacionados com retenção hídrica são frequentes e causam congestão pulmonar (estertores pulmonares), elevação de pressão venosa (estase de veias jugulares) e edema periférico. ${ }^{4}$

O diagnóstico de IC é fundamentalmente clínico, mas podem-se empregar critérios para torná-lo menos subjetivo. Para o diagnóstico da IC, os critérios de Framingham, valorizam estes sinais e sintomas:

- critérios maiores: dispneia paroxística noturna, distensão das veias do pescoço, estertores pulmonares, cardiomegalia à radiografia de tórax, edema pulmonar agudo, terceira bulha, pressão venosa central aumentada $>16 \mathrm{cmH}_{2} \mathrm{O}$, tempo de circulação $\geq 25$ segundos, refluxo hepatojugular, edema pulmonar, congestão visceral ou cardiomegalia na autópsia, perda de peso $\geq 4,5 \mathrm{Kg}$ em cinco dias em resposta ao tratamento da IC.

- critérios menores: edema de tornozelo bilateral, tosse noturna, dispneia às atividades habituais, hepatomegalia, derrame pleural, diminuição da capacidade vital em 1/3 do valor máximo alcançado, taquicardia com frequência cardía$\mathrm{ca} \geq 120 \mathrm{bpm} .{ }^{22}$

A anemia na IC causa exacerbação desses sinais e sintomas, principalmente da dispneia, fadiga, edema e isquemia. ${ }^{19}$ Nos pacientes com disfunção sistólica, a presença de anemia está associada à classe funcional (NYHA) mais avançada, ${ }^{14,23}$ capacidade menor de exercício, ${ }^{23,24,25}$ menor distância percorrida no teste de caminhada de seis minutos, pico de consumo de $\mathrm{O}_{2}$ mais baixo durante teste cardiopulmonar, ${ }^{23,25}$ função renal mais deteriorada, ${ }^{14,23}$ progressão mais rápida da insuficiência renal, maior grau de hipotensão arterial, taquicardia e pressão capilar pulmonar mais elevada. ${ }^{23}$

$\mathrm{Na}$ disfunção diastólica, a presença de anemia está associada com aumento de mortalidade, aumento de hospitalizações, níveis maiores de BNP e redução da capacidade de exercício. ${ }^{18,24,26}$ Muitos estudos demonstraram que a anemia é um importante preditor independente de mortalidade e de hospitalizações em pacientes com IC sistólica ou diastólica. ${ }^{18,26}$ Horwich et al. demonstraram que, em pacientes com IC avançada, a cada decréscimo de $1 \mathrm{~g} / \mathrm{dl}$ de Hb há um aumento no risco de morte em $13 \%{ }^{23} \mathrm{Na}$ mesma linha de pesquisa, Mozaffarian et al. demonstraram que, em pacientes com IC avançada, a redução do $\mathrm{Ht}$ de $1 \%$ causa aumento do risco de morte em $11 \% .{ }^{27}$

Em outro estudo realizado no Hospital de Cotoxó InCor (anos 2005 e 2006), com 263 pacientes internados com IC e disfunção sistólica (FE $<45 \%$ ), foi avaliada a evolução do paciente conforme a presença de anemia na internação e durante o acompanhamento ambulatorial após a alta. Este estudo revelou que $21,6 \%$ dos pacientes permaneceram com anemia, 9,9\% deixaram de apresentar anemia durante o acompanhamento ambulatorial e $18,7 \%$ dos pacientes que não apresentavam anemia na hospitalização passaram a apresentá-la. É interessante salientar que não houve tratamento específico para anemia, e a variação encontrada nos níveis de hemoglobina esteve unicamente relacionada ao tratamento usual da IC. A evolução dos pacientes diferiu conforme a evolução da anemia no seguimento, sendo a taxa de mortalidade de $7,1 \%, 11,8 \%, 24,3 \%$ e $34,4 \%$ respectivamente para os que nunca apresentaram anemia, para aqueles em que a anemia regrediu, naqueles com anemia persistente e nos pacientes em que a anemia apareceu (Figura 2). A taxa de mortalidade foi significativamente maior para os pacientes com anemia do que para os que nunca tiveram anemia ou em que ela regrediu. Nos pacientes em que a anemia melhorou houve uma evolução semelhante à dos que nunca apresentaram anemia durante o seguimento.

\section{Abordagem diagnóstica}

O diagnóstico da IC é clínico e deve ser baseado na avaliação de sinais e sintomas. ${ }^{1}$ Os critérios de Framingham são úteis para o diagnóstico da IC, sendo necessário o acha- 


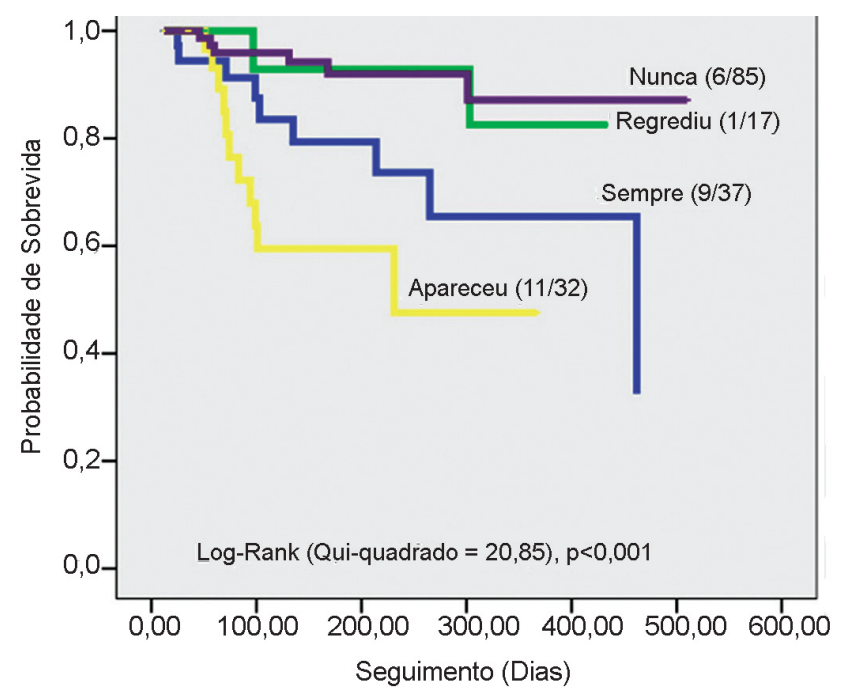

Figura 2. Curva de sobrevida em relação à evolução da anemia entre a internação e o seguimento. Grupos: Nunca (sem anemia na internação e no seguimento); Regrediu (anemia na internação e sem anemia no seguimento); Sempre (anemia persistente, tanto na internação quanto no seguimento); Apareceu (sem anemia na internação, mas com anemia no seguimento)

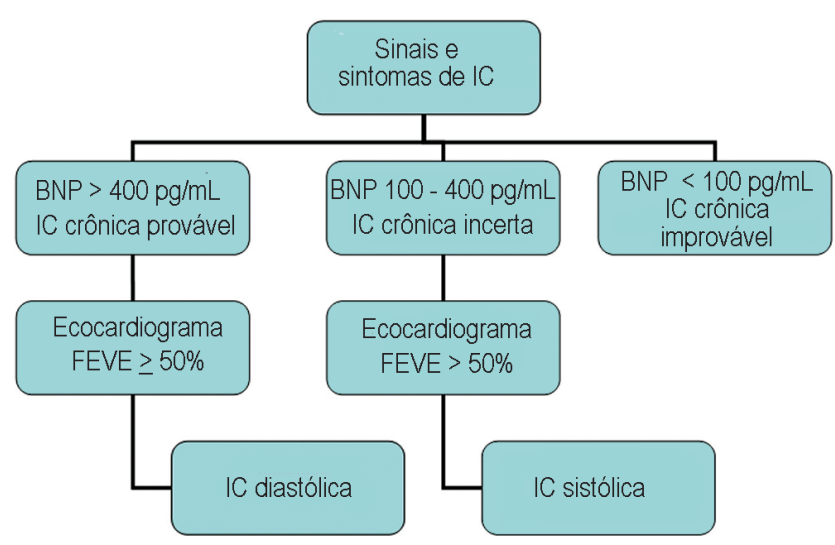

Figura 3. Fluxograma de diagnóstico de IC crônica adaptado da III Diretriz Brasileira de insuficiência cardíaca crônica 2009. ${ }^{7}$

FEVE = Fração de ejeção do ventrículo esquerdo

do de dois critérios maiores e um menor ou dois menores e um maior.

Dentre os exames complementares úteis no diagnóstico e seguimento dos pacientes portadores dessa síndrome estão a radiografia de tórax, o eletrocardiograma, o ecocardiograma bidimensional com Doppler e, mais recentemente, a dosagem do BNP (peptídeo natriurético tipo B). Este peptídeo é produzido pelos ventrículos e tem se mostrado útil na avaliação diagnóstica do paciente com suspeita de IC. ${ }^{7} \mathrm{Na}$ Figura 3 apresentamos um fluxograma para diagnóstico de insuficiência cardíaca. ${ }^{1,7}$

Para pesquisa de anemia, deve ser solicitado hemograma completo e, caso confirmado, devemos persistir na investi- gação da etiologia. Portanto, é necessário dosagem de ferro sérico, saturação de transferrina e ferritina sérica na investigação da etiologia. ${ }^{28} \mathrm{O}$ ácido fólico e vitamina B12 também devem ser dosados. ${ }^{29}$

\section{Prevenção e tratamento}

Não há estudos ou consensos que mostrem medidas para prevenção da anemia na IC. Porém, podemos adotar as mesmas medidas preventivas empregadas no controle das anemias de doença crônica, deficiência de ferro e outros minerais.

Diante de tantos estudos reconhecendo a anemia como comorbidade prevalente no portador de disfunção sistólica ou diastólica e como preditor independente de mortalidade e hospitalizações, a ideia de tratá-la é atrativa porque sabemos que é uma causa de exacerbação das manifestações clínicas e associada à pior evolução da IC. Entretanto, até o presente momento não há evidência que mostre o real benefício da correção do nível de hemoglobina nessa síndrome. ${ }^{19}$

Entre as propostas terapêuticas devemos considerar o suplemento de ferro isolado e a administração de agentes estimulantes da eritropoetina, como seu análogo darbepoetina. ${ }^{30}$ Bolger et al., em um estudo não randomizado no tratamento da anemia de pacientes com IC, usando ferro isolado, trataram 16 pacientes com $\mathrm{Hb}<12$, CF II-III NYHA e ferro sérico $<400 \mathrm{ng} / \mathrm{mL}$. A deficiência de ferro estava presente em $44 \%$ dos pacientes, usando critério diagnóstico de Tsat, $16 \%$ e ferritina $<30 \%$. Os pacientes foram tratados com reposição de ferro intravenosa por 12 dias e seguidos por $92 \pm 6$ dias. Este estudo revelou um aumento de $\mathrm{Hb}$ de $11,2 \mathrm{~g} / \mathrm{dL} \pm 0,7$ para $12,6 \mathrm{~g} / \mathrm{dL} \pm 1,2$, melhora de classe funcional NYHA e do teste de caminhada de seis minutos. Mas a resposta foi inadequada quando foi usado apenas o ferro isolado, sem o uso do estimulante de eritropoetina. ${ }^{31}$ No entanto, esta conclusão é limitada pelo número pequeno de pacientes estudados e pela sua seleção não randomizada.

A reposição de ferro também foi testada no estudo Ferric-HF, ${ }^{32}$ onde foi avaliado o uso do ferro endovenoso na melhora de capacidade de exercício em 35 pacientes deficientes de ferro (ferritina $<100 \mathrm{ug} / \mathrm{L}$ ou ferritina entre 100-300 e Sat $\mathrm{T}<20 \%$ ). Foi um estudo randomizado, placebo controlado, que revelou que o grupo tratamento $(200 \mathrm{mg}$ de ferro/semana durante quatro semanas (e a seguir $200 \mathrm{mg} / \mathrm{mês}$ durante três meses de manutenção) obteve melhora dos níveis de ferro e de classe funcional em comparação ao grupo placebo. Diferente dos achados de Bolger, não houve diferença na concentração de $\mathrm{Hb}$ entre o grupo que recebeu tratamento e o placebo. ${ }^{31,32}$ Estudos pequenos sugerem que o tratamento com ferro isolado reduz o BNP de pacientes com IC e insuficiência renal. ${ }^{33} \mathrm{O}$ estudo FAIR-HF, publicado recentemente, avaliou pacientes com IC sistólica, classe funcional II e III (NYHA) e deficiência de ferro associada (Ferritina $<100 \mathrm{mcg} / \mathrm{L}$ ou ferritina 100-299 mcg/L e saturação de transferrina $<20 \%$ ), 
em pacientes com hemoglobina entre 9,5 a 13,5 g/dL. Foi um estudo randomizado e prospectivo, onde os pacientes receberam ferro endovenoso ou placebo. O resultado revelou que o tratamento com ferro endovenoso em pacientes com IC e deficiência de ferro, na presença ou não de anemia, melhorou os sintomas, a capacidade funcional e a qualidade de vida. ${ }^{34}$ Em relação aos agentes estimulantes de eritropoese, $o$ ensaio clínico disponível sobre esta proposta resultou em melhora dos níveis de hemoglobina, porém sem evidências de benefício no tempo de exercício.?

Com relação ao tratamento da anemia, as diretrizes brasileiras de IC crônica ${ }^{7}$ consideram:

- reposição de ferro oral ou venoso para correção de anemia ferropriva $(\mathrm{Hb}<12 \mathrm{~g} / \mathrm{dL})$ como classe I de recomendação e nível de evidência $C$;

- administração de eritropoetina e ferro venoso para correção de anemia $(\mathrm{Hb}<12)$ em pacientes com insuficiência renal crônica e IC como classe de recomendação I e nível de evidência $B$.

- transfusões de sangue em pacientes anêmicos $(\mathrm{Hb}<9)$ com cardiomiopatia isquêmica ou pacientes anêmicos com cardiomiopatia dilatada de etiologia não isquêmica como classe de recomendação IIa e nível de evidência C.

É importante ressaltar que apenas com o tratamento específico da insuficiência cardíaca há uma melhora dos níveis de hemoglobina, como observado em nossa experiência. O desaparecimento da anemia sem um tratamento específico chama a atenção para a possibilidade de a anemia ser um epifenômeno na IC e sua presença ser somente um sinal de que o paciente possui uma doença mais grave. Com a compensação da IC, os fatores causais da anemia, como a inflamação, a inapetência (redução da ingestão de ferro e vitaminas) e a redução da perfusão renal, melhoram e a anemia pode desaparecer. Por outro lado, não podemos deixar de considerar que o tratamento da anemia corrigindo a depleção de ferro e a deficiência relativa ou absoluta de eritropoetina possa melhorar a evolução dos pacientes.

A reposição de ferro e eritropoetina podem ser feitas conforme esquema abaixo:

\section{Reposição do ferro}

$\mathrm{O}$ tratamento com ferro deve ser feito quando há níveis de depósito de ferro reduzido. Devemos lembrar que muitos pacientes podem ter má absorção de ferro ou ser intolerante ao medicamento oral, e por esses motivos damos preferência ao tratamento endovenoso. Devemos prescrever o ferro endovenoso se houver presença de anemia, associada a deficiência de ferro (ferritina $<100 \mathrm{ng} / \mathrm{ml}$ e Sat transferrina $<20 \%$ ) e com taxa de filtração glomerular $<60 \mathrm{~mL} / \mathrm{min}$. O esquema geralmente utilizado é a reposição com uma ampola de ferro por semana durante dez semanas, ou duas ampolas a cada duas semanas durante cinco semanas. Deve ser feita a avaliação dos índices de $\mathrm{Hb} / \mathrm{Ht}$ mensalmente e a dosagem da reserva de ferro a cada três meses. Uma fórmula prática que utilizamos para encontrar a dose total de ferro $(\mathrm{em} \mathrm{mL})$ é a seguinte:

$$
\mathrm{N}(\mathrm{ml})=(\text { Peso em Kg } \times \text { Dhb } \times 2,4)+500 \mathrm{mg}
$$

20

Onde $\mathrm{N}$ = quantidade de ferro em $\mathrm{ml}$ a ser administrado endovenoso

$\mathrm{Dhb}=$ diferença entre a hemoglobina desejada e a encontrada

$500 \mathrm{mg}=$ reserva necessária de ferro

\section{Reposição de eritropoetina}

Deve ser prescrita se não houver deficiência de ferro ou com sobrecarga de ferro. Aplicar eritropoetina por via subcutânea com periodicidade semanal, devendo ser realizada dosagem de hemoglobina mensalmente e índice de ferro a cada três meses.

Ainda faltam grandes estudos que evidenciem a redução da morbi/mortalidade associada ao tratamento específico da anemia na IC. Persistem dúvidas sobre valor da reposição de ferro, sobre a segurança do tratamento da eritropoetina e se a combinação de ambas seria sempre necessária para o melhor controle da anemia nos nossos pacientes. Entretanto, anemia deve ser investigada e valorizada nos portadores de insuficiência cardíaca e, provavelmente, em breve, será sistematicamente tratada visando seu controle e consequente melhora na evolução de nossos pacientes.

\begin{abstract}
Anemia is common in heart failure (HF) patients with its presence apparently associated to a worse prognosis, and as such is described in some studies as an independent predictor of death and hospitalization of patients suffering from systolic and diastolic dysfunction. It remains unknown whether anemia causes the worse evolution of HF patients or whether it is only one marker of a worse heart disease stage. The etiology of anemia is multifactorial and seems to change dependent on the studied population. Factors such as nutritional iron deficiency, presence of kidney failure, intense systematic inflammatory activity, medication use that inhibits the production of erythropoietin or that results in blood loss are the most frequent causes of anemia in heart disease. The prevalence of anemia reported in studies of HF is very variable (from 9 to $79.1 \%$ ) and is dependent of the studied population, stage of heart disease and method and references used for diagnosis. Iron deficiency is an important etiologic factor which is present in a significant number of patients with the association of anemia and HF. In a study carried out by our group, the incidence of iron deficiency in anemic patients was $61.8 \%$. Hence, anemia is a frequent finding; its presence accentuates the clinical manifestations of $\mathrm{HF}$ and is associated to a worse prognosis. An understanding of the cause of anemia makes treatment easier and although there is no consensus on its correction, patients without anemia have a better evolution. Rev. Bras. Hematol. Hemoter. 2010;32(Supl.2):89-94.
\end{abstract}

Key words: Heart failure; anemia; iron deficiency. 


\section{Referências Bibliográficas}

1. Barretto ACP. Insuficiência cardíaca descompensada. Ed Phoenix, $1^{a}$ edição, 2007:7-26,93-120.

2. Braunwald E. Heart Failure - A text book of cardiovascular medicine. Fisiopatologia da insuficiência cardíaca, $7^{\text {th }}$ edition, Elsevier Saunders, 2005:509-38.

3. Okonko DO, Anker SD. Anemia in chronic heart failure: pathogenic mechanisms. J Card Fail. 2004;10(1 Suppl):S5-9.

4. Givertz MM, Colucci WS,Braunwald E. Clinical aspects of heart failure: high-output failure; pulmonary edema. In: Braunwald' Heart Disease: a textbook of cardiovascular medicine, $7^{\text {th }}$ edition, Elsevier Saunders, 2005:539-68.

5. Maggioni AP, Opasich C, Anand I, Barlera S, Carbonieri E, Gonzini $\mathrm{L}$, et al. Anemia in patients with heart failure: prevalence and prognostic role in a controlled Trial and in clinical practice. J. Card Fail. 2005;11(2):91-8.

6. McMurray JJ. What are the clinical consequences of anemia in patients with chronic heart failure? J Card Fail. 2004;10(1 Suppl): S10-2.

7. III Diretriz Brasileira de Insuficiência Cardíaca Crônica. Arq Bras Cardiol. 2009;93(1 supl.1):1-71.

8. Serrano C. Tratado de Cardiologia Socesp. Ed Manole, $2^{\mathrm{a}}$ edição, 2010:1019-27.

9. Tang YD, Katz SD. Anemia in chronic heart failure: prevalence, etiology, clinical correlates, and treatment options. Circulation 2006;23;113(20):2454-61.

10. Go AS, Yang J, Ackerson LM, Lepper K, Robbins S, Massie BM, et al. Hemoglobin level, chronic disease, and the risks of death and hospitalization in adults with chronic heart failure: Anemia in Chronic Heart Failure: Outcomes and Resource Utilization (ANCHOR) Study. Circulation. 2006; 13:113(23):2713-23.

11. Komajda M. Prevalence of anemia in patients with chronic heart failure and their clinical characteristics. J Card Fail. 2004;10(1 Suppl):S1-4.

12. Sales AL, Villacorta H, Reis L, Mesquita ET. Anemia as a prognostic factor in a population hospitalized due to descompensated heart failure. Arq Bras Cardiol. 2005;8 4(3): 237-40.

13. Horowich TB, Fonarow GC, Hamilton MA, MacLellan WR, Borenstein J. Anemia is associated with worse symptoms, greater impairment in functional capacity and a significant increase in mortality in patients with advanced heart failure. J. Am Coll Cardiol. 2002;5:39(11):1780-6

14. Silverberg DS, Wexler D, Blum M, Keren G, Sheps D, Leibovitch E, et al. The use of subcutaneous erythropoietin and intravenous iron for the treatment of the anemia of severe, resistant congestive heart failure improves cardiac and renal function and function cardiac class, and markedly reduces hospitalizations. J Am Coll Cardiol. 2000;35(7):1737-44.

15. Ralli S, Horwich TB, Fonarow GC Relationship between anemia, cardiac troponin I, and B-type natriuretic peptide levels and mortality in patients with advanced heart failure. Am Heart J. 2005;150(6)1220-7

16. Mitchell JE. Emerging role of anemia in heart failure. Am J Cardiol. 2007;99:15D-20D.

17. Al-Ahmad A, Rand WM, Manjunath G, Konstam MA, Salem DN, Levey AS, et al. Reduced kidney function and anemia as risk factors for mortality in patients with left ventricular dysfunction. J Am Coll Cardiol. 2001;38(4):955-62.

18. Felker GM, Shaw LK, Stough WG, O'Connor CM. Anemia in patients with heart failure and preserved systolic function. Am Heart J. 2006;151(2):457-62.

19. Murphy CL, McMurray JJ. Approaches to the treatment of anaemia in patients with chronic heart failure. Heart Fail Rev. 2008; 13(4):431-8.

20. Olivetti G, Lagrasta C, Quaini F, et al. Capillary growth in anemiainduced ventricular wall remodeling in the rat heart. Circ Res. 1989;65(5):1182-92.

21. Levin A, Thompson CR, Ethier J, Carlisle EJ, Tobe S, Mendelssohn $\mathrm{D}$, et al. Left ventricular mass index increase in early renal disease: impact of decline in hemoglobin. Am J Kidney Dis. 1999; 34(1):125-34

22. Ho KK, Pinky J, Kannel WB, et al. The epidemiology of heart failure: The Framingham Study. J Am Coll Cardiol. 1993:22:6A-13A.

23. Horwich TB, Fonarow GC, Hamilton MA, MacLellan WR, Borenstein J. Anemia is associated with worse symptoms, greater impairment in functional capacity and a significant increase in mortality in patients with advanced heart failure. J Am Coll Cardiol. 2002;39 (11):1780-6.

24. Witte KK, Desilva R, Chattopadhyay S, Ghosh J, Cleland JG, Clark AL. Are hematinic deficiencies the cause of anemia in chronic heart failure. Am Heart J. 2004;147(5):924-30.

25. Mancini DM, Katz SD, Lang CC, LaManca J, Hudaihed A, Androne AS. Effect of erythropoietin on exercise capacity in patients with moderate to severe chronic heart failure. Circulation. 2003;107 (2):294-9.

26. O'Meara E, Clayton T, McEntegart MB, McMurray JJ, Lang CC, Roger SD, et al. Clinical correlates and consequences of anemia in a broad spectrum of patients with heart failure: results of the Candesartan in Heart Failure: Assessment of Reduction in Mortality and Morbidity (CHARM) Program. Circulation. 2006; 113(7):986-94.

27. Mozaffarian D, Nye R, Levy WC. AAnemia predicts mortality in severe heart failure: the prospective randomized amlodipine survival evaluation (PRAISE). J Am Coll Cardiol. 2003;41(11):1933-9.

28. Weiss G, Goodnough LT. Anemia of chronic disease. N Engl J Med. 2005;352(10):1011-23.

29. Drakos SG, Anastasiou-Nana MI, Malliaras KG, Nanas JN. Anemia in chronic heart failure. Congest Heart Fail. 2009;15(2):87-92.

30. Bolger AP, Bartlett FR, Penston HS, O'Leary J, Pollock N, Kaprielian $\mathrm{R}$, et al. Intravenous iron alone for the treatment of anemia in patients with chronic heart failure. J Am Coll Cardiol. 2006; 48(6):1225-7.

31. Ghali JK, Anand IS, Abraham WT, Fonarow GC, Greenberg B, Krum H, et al; on behalf of the Study of Anemia in Heart Failure Trial (STAMINA-HeFT) Group 2008; Randomized double-blind trial of darbepoetin alfa in patients with symptomatic heart failure and anemia. Circulation. [Epub ahead of print]

32. Toblli JE, Lombraña A, Duarte P, Di Gennaro F. Intravenous iron reduces NT-pro-brain natriuretic peptide in anemic patients with chronic heart failure and renal insufficiency. J Am Coll Cardiol. 2007;50(17):1657-65.

33. Anker SD, Cornin Colet JC, Filippatos G, Willenheimer R, Dickstein $\mathrm{K}$, Drexler H, et al. Ferric carboxymaltose in patients with heart failure and iron deficiency. N Engl J Med. 2009;361(25):2436-48.

O tema foi sugerido e avaliado pelo coeditor deste fascículo educativo, Rodolfo Delfini Cançado, e pelo board interno da RBHH, e publicado após a concordância do editor, Milton Artur Ruiz.

Conflito de interesse: sem conflito de interesse

Recebido: 20/12/2009

Aceito: 15/01/2010 